\title{
A strategic decision support system for logistics and supply chain network design
}

\author{
T BISWAS* and SUSMITA SAMANTA \\ Department of Computer Science and Engineering, St. Thomas' College of Engineering and Technology, \\ Kolkata 700023, India \\ e-mail: amitaimika@gmail.com
}

MS received 25 November 2015; revised 22 January 2016; accepted 1 February 2016

\begin{abstract}
This paper aims to develop a strategic decision support system for logistics and supply chain network design of a multi-stage, multi-commodity, and multi-period distribution and transportation system. A mixed integer linear programming model is proposed to tackle the problem while minimizing the operating, transportation and handling cost through all tiers of the supply chain network. A genetic algorithm based method has been proposed to solve the problem in a large scale realistic environment. The efficacy of the developed strategic decision support model in achieving better utilization of network and resources to fulfil the customer demand is demonstrated using illustrative scenarios inspired from the real case of a logistics company.
\end{abstract}

Keywords. Supply chain network; logistics network; facility location; decision support system; CPLEX; genetic algorithm.

\section{Introduction}

In the present competitive era, logistics and supply chain network plays a vital role towards the economic growth of a country. Manufacturing companies focus on developing a logistics network to fulfil the customer demand by taking account of the market demand. Due to this, in the last decade, the role of manufacturing has shifted from simple producers of goods and services, to ones that co-ordinate the whole industry value chain. Now, manufacturers have own activities to integrate all intermediate activities to provide the product to the customers without any delay. Network design decisions have a significant impact on performance, because they determine the supply chain configuration and set constraints within which the other supply chain drivers can be used to decrease the supply chain cost or increase responsiveness. All network design decisions affect one another and must be made taking into this consideration. Decisions concerning the role of each facility are significant, because they determine the amount of flexibility the supply chain network has in changing the way, it meets demand.

A supply chain distribution network as shown in figure 1 describes three stages and four levels of network. The two levels of entities based on customer-supplier relationship have been considered in a generic framework of network design. The available levels are the sources, named also manufacturing plants (at the first level), the central

*For correspondence distribution centres (CDCs, at the second level), the regional distribution centres (RDCs) representing the third level, and finally the customers demand points (CDPs) at the fourth level. This is an example of a generic distribution network and this configuration is adopted in this paper and treated by the proposed models, methods and DSS as discussed in the rest of the paper.

A decision support system is now developed to design and control the multi-echelon logistics distribution network at different level of supply chain systems. A genetic algorithm (GA) based method is proposed to support the decision making process on strategic and operational planning. This model can be used to design and control many real and complex practical scenarios and can be used by practitioners, planners and managers easily.

The remainder of this paper is organized as follows. The literature review is described in section 2. Section 3 presents problem description and the mathematical model. The methodology and approach is described in section 4 . We summarize the results and discussion in section 5. Finally, conclusion is presented in section 6 .

\section{Literature review}

A model for multi-product, multi-period three stage supply chain network with warehouse reliability is developed by Pasandideh et al [1]. A bi-objective mixed integer linear programming is formulated to minimize the total cost of network used for supply chain and maximize the total 


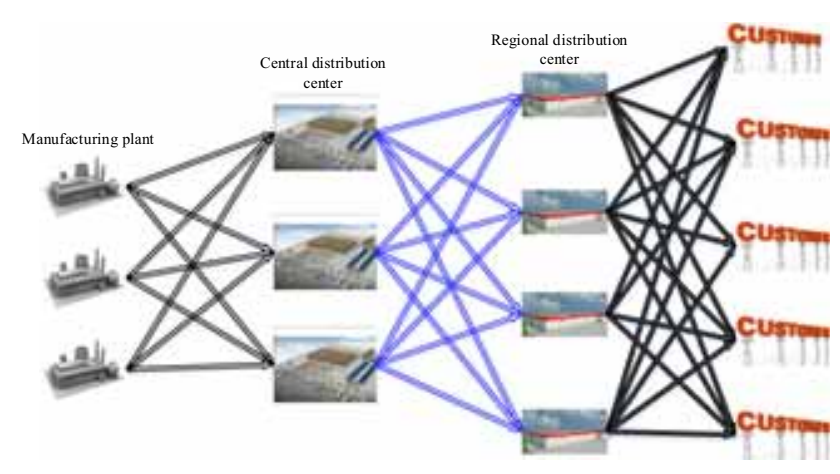

Figure 1. A supply chain distribution network.

number of products dispatched to the customer [2]. In recent growth of freight transportation, the greenhouse gas emission influences the factors of sustainable supply chain network. He et al [3] introduced a problem on multi-echelon container supply chain and modeled as mixed integer linear programming to minimize the total supply chain service cost. The closed loop supply chain for multi-product, muti-echelon model has been described by Kalaitzidou et al [4] to fulfil the market demand by minimizing the overall capital and operational cost. A closed loop supply chain model is developed to capture the environmental issues [5] and determine the flow of parts and products in CLSC network. A rule based optimization method is proposed by Pratap et al [6] for bulk handling port operations. Fattahi et al [7] developed a model for multi-echelon and multi-product supply chain network for multi-period horizon and maximize the profit and revenue in network.

The design of sustainable supply chain network is described by Dejax and Olivier [8], who present various sustainable factors influencing the supply chain network. Mousavi et al [9] designed a model for supply chain network for seasonal multiple product location/allocation inventory problem and minimize the inventory cost associated with distributor and retailer. A reconfigurable supply chain network is developed by Kristianto et al [10] which minimize the inventory allocation and transportation routing cost. Pramanik et al [11] presented a model for two stage supply chain network with imprecise capacitated fixed charge transportation cost and maximize the profit in order to fulfil the retailers demand with associated distributed centre. Mousavia et al [12] designed a supply chain network model for sessional multiple product allocation for inventory management in a certain time horizon.

\section{Problem statement}

The logistics supply chain network design and distribution network are mainly dependent on strategic planning of large enterprises company. Simchi-Levi et al [13] describes

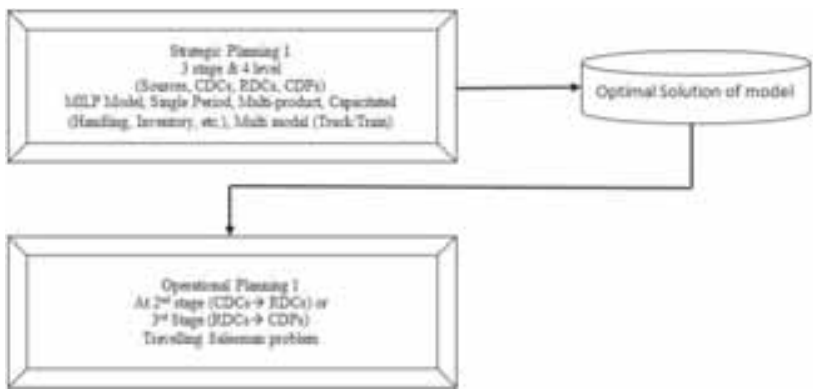

Figure 2. Logistics network to design the strategy model.

the long effect influence of the strategic level decision on a company. Supply chain networks should be managed by taking into consideration the objectives of all supply chain stakeholders [14].

The design of logistics network controls the multi-echelon logistics production distribution network. For designing, decision making should be based on strategic planning and operational planning.

Strategic level planning is generally classified on the basis of the following:

- Capacity decision

- Inventory decision

- Procurement decision

- Production decision

- Routing decision

- Choice of transportation model

In the model, the complexities involved in designing the network are dimension of instance (number of entities-DCs, production plant, customer, etc.), products (single vs multicommodity) and planning period (existing of single sourcing hypothesis), as shown in figure 2 .

\subsection{Mathematical model}

3.1a Objective: To determine the optimal location of production/distribution centres, allocation of customer demand (activation of links and flows between two generic entities of different levels e.g. central DCs and regional DCs) and determine the optimal handling and storage capacities of available set of production/distribution centres.

Index

$$
\begin{aligned}
& p=\text { production plant }\{p=1,2,3 \ldots \mathrm{P}\} \\
& c=\text { central distribution centre }\{c=1,2,3 \ldots \mathrm{C}\} \\
& q=\text { regional distribution centre }\{q=1,2,3 \ldots \mathrm{Q}\} \\
& t=\text { transportation mode }\{t=1,2,3 \ldots \mathrm{T}\} \\
& i=\text { customer demand point }\{i=1,2,3 \ldots \mathrm{I}\} \\
& l=\text { number of products }\{l=1,2,3 \ldots \mathrm{L}\}
\end{aligned}
$$

where $\mathrm{M}, \mathrm{D}$ and $\mathrm{R}$ represent a manufacturing plant, central distribution centre, and regional distribution centre, respectively. 


\section{Decision variables}

$f_{p}^{M} \quad$ fixed cost for manufacturing plant $p$

$f_{c}^{D} \quad$ fixed cost for central distribution centre $c$

$f_{q}^{R} \quad$ fixed cost for regional distribution centre $q$

$y_{p} \quad \begin{cases}1 & \text { if plant } p \text { is used } \\ 0 & \text { otherwise }\end{cases}$

$y_{c} \quad \begin{cases}1 & \text { if distribution centre } c \text { is used } \\ 0 & \text { otherwise }\end{cases}$

$y_{q} \quad \begin{cases}1 & \text { if regional distribution centre } q \text { is used } \\ 0 & \text { otherwise }\end{cases}$

$x_{p c t l} \quad$ Quantity of product $l$ flows from plant $p$ to distribution centre $c$ using transportation mode $t$

$x_{\text {cqtl }} \quad$ Quantity of product $l$ flows from distribution centre $c$ to regional distribution centre $q$ using transportation mode $t$

$x_{\text {qitl }} \quad$ Quantity of product $l$ flows from regional distribution centre $q$ to customer demand point using transportation mode $t$

$C_{p c t l}^{s} \quad$ Transportation cost of product $l$ per unit load from plant $p$ to central distribution centre $c$ through transportation mode $t$

$C_{p q t l}^{c} \quad$ Transportation cost of product $l$ per unit load from central distribution centre $c$ to regional distribution centre $q$ through transportation mode $t$

$C_{q i t l}^{c} \quad$ Handling cost of product $l$ from regional distribution centre $q$ to customer order demand point $i$ through transportation mode $t$

$v_{p l}^{M} \quad$ Variable cost per load from manufacturing plant $p$ of product $l$

$v_{c l}^{D} \quad$ Variable cost per load from distribution centre $c$ of product $l$

$v_{q l}^{R} \quad$ Variable cost per load from regional distribution centre $q$ of product $l$

\section{Strategy planning}

$$
\begin{aligned}
\theta= & \sum_{p=1}^{P} f_{p}^{M} \cdot y_{p}+\sum_{c=1}^{C} f_{c}^{D} \cdot y_{c}+\sum_{q=1}^{Q} f_{q}^{R} \cdot y_{q} \\
& +\sum_{p=1}^{P} \sum_{c=1}^{C} \sum_{t=1}^{T} \sum_{l=1}^{L} C_{p c t l}^{s} \cdot x_{p c t l}+\sum_{c=1}^{C} \sum_{q=1}^{Q} \sum_{t=1}^{T} \sum_{l=1}^{L} C_{p q t l}^{c} \cdot x_{p q t l} \\
& \sum_{q=1}^{Q} \sum_{i=1}^{M} \sum_{t=1}^{T} \sum_{l=1}^{L} C_{q i t l}^{c} \cdot x_{q t l}+\sum_{p=1}^{P} \sum_{c=1}^{C} \sum_{t=1}^{T} \sum_{l=1}^{L} x_{p c t l} \cdot v_{p l}^{M} \\
& +\sum_{q=1}^{Q} \sum_{p=1}^{P} \sum_{t=1}^{T} \sum_{l=1}^{L} x_{p q t l} \cdot v_{c l}^{D}+\sum_{q=1}^{Q} \sum_{i=1}^{I} \sum_{l=1}^{L} x_{q i l} \cdot v_{q l}^{R}
\end{aligned}
$$

$\min \{\theta\}$

Fixed cost fixed cost for manufacturing plant

$$
\sum_{c=1}^{C} f_{c}^{D} \cdot y_{c}
$$

fixed cost for central distribution centre

$$
\sum_{q=1}^{Q} f_{q}^{R} \cdot y_{q}
$$

fixed cost for regional distribution centre

Transportation cost

$$
\sum_{p=1}^{P} \sum_{c=1}^{C} \sum_{t=1}^{T} \sum_{l=1}^{L} C_{n p t l}^{s} \cdot x_{n p t l} .
$$

Manufacturing plant to distribution centre $(p)$

$$
\sum_{c=1}^{C} \sum_{q=1}^{Q} \sum_{t=1}^{T} \sum_{l=1}^{L} C_{p q t l}^{c} \cdot x_{p q t l} .
$$

Distribution centre $(p)$ to regional distribution centre $(q)$

$$
\sum_{q=1}^{Q} \sum_{i=1}^{M} \sum_{t=1}^{T} \sum_{l=1}^{L} C_{q i t l}^{c} \cdot x_{q t l} .
$$

Regional distribution centre $(q)$ to Customer point order Handling cost

$$
\sum_{p=1}^{P} \sum_{c=1}^{C} \sum_{t=1}^{T} \sum_{l=1}^{L} x_{n p t l} \cdot v_{n l}^{M} .
$$

Handling cost to supply product $l$ from plant $n$ to distribution centre $(p)$ by transportation $a$

$$
\sum_{q=1}^{Q} \sum_{p=1}^{W} \sum_{t=1}^{T} \sum_{l=1}^{L} x_{p q t l} \cdot v_{p l}^{D} .
$$

Handling cost to supply product $l$ from distribution centre $(p)$ to redistribution center by route $a$

$$
\sum_{q=1}^{Q} \sum_{i=1}^{W} \sum_{l=1}^{L} x_{q i l} \cdot v_{q l}^{R} .
$$

Handling cost to supply product $l$ from redistribution center $(q)$ to customer order point

$$
\sum_{q=1}^{Q} x_{q i l}=d_{i l} .
$$

Total quantity of product $l$ flows from RDCs to demand point $i$

$$
\sum_{c=1}^{C} \sum_{t=1}^{T} x_{p c t l} \leq h C_{p l}^{M} y_{p}
$$


Total quantity of product $l$ from plant $p$ to distribution centre $c$ through transportaion mode $t$ is greater than supply/production capacity of source plant $p$

$$
\sum_{q=1}^{Q} \sum_{t=1}^{T} x_{c q t l} \leq h C_{q l}^{D} y_{c}
$$

Total quantity of product $l$ from distribution centre $c$ to regional distribution centre $q$ through transportation mode $t$ is greater than supply/production capacity of distribution centre $c$

$$
\sum_{i=1}^{Q} \sum_{t=1}^{T} x_{q i t} \leq h C_{i l}^{R} y_{q}
$$

Total quantity of product $l$ from distribution centre $c$ to regional distribution centre $q$ to customer demand point $i$ is greater than supply/production capacity of regional distribution $q$.

\section{Methodology}

The following two methods are used to solve the problem.

\subsection{Genetic algorithm}

A nature inspired genetic algorithm is proposed to solve the mixed integer linear programming (MILP) problem to determine the optimal strategy. Genetic algorithms (GAs) are adaptive heuristic search algorithms based on the evolutionary ideas of natural selection and genetics as described in figure 3. As such, they represent an intelligent exploitation of a random search used to solve optimization problems. Although randomized, GAs are by no means random, instead they exploit historical information to direct the search into the region of better performance within the search space.

\section{Crossover}

Crossover is the main genetic operator. It operates on two parents (chromosomes) at a time and generates offspring by combining both chromosomes' features. The crossover is done to explore new solution space and crossover operator corresponds to exchanging parts of strings between selected parents.

\section{Mutation}

Mutation is another important operator which generates random changes in chromosomes. Similar to crossover, mutation is done to prevent the premature convergence and explore new solution space. However, unlike crossover, mutation is usually done by modifying gene within a chromosome. We also investigate the effects of two different mutation operators on the performance of GA. Insert mutation is used for this purpose. Several mutation operators have been proposed for permutation representation,

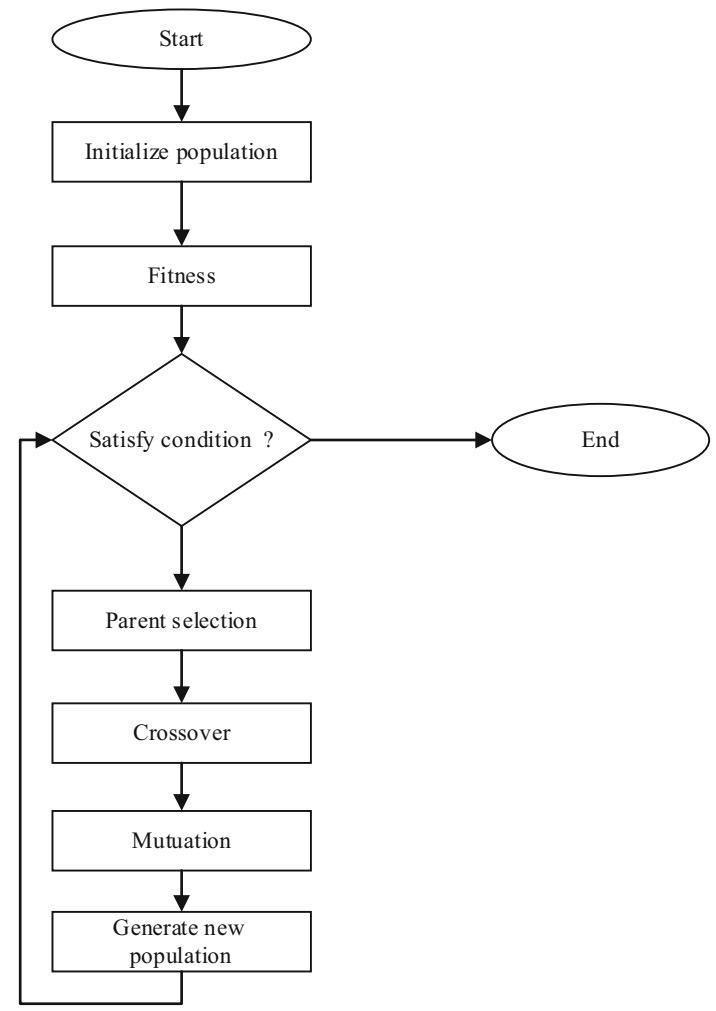

Figure 3. Genetic algorithm to evaluate fitness function.

such as inversion, insertion, displacement, and reciprocal exchange mutation.

\section{Evaluation of fitness}

The evaluation aims to associate each individual with a fitness value, so that it can reflect the goodness of fit for an individual. The evaluation process is intended to compare one individual with other individuals in the population. The choice of fitness function is also very critical, because it has to accurately measure the desirability of the features described by the chromosome. The function should be computationally efficient, since it is used many times to evaluate each solution. In this study, the objective function has been taken as fitness function.

\subsection{ILOG CPLEX optimizer}

ILOG CPLEX optimizer is a flexible and user-friendly software platform for manufacturing plant in logistics sections, encountering the multi-echelon and multi-level enterprises. It creates a platform to integrate development environment with optimization programming language (OPL) and can be implemented in recent Microsoft environments It is flexible because the strategic planning and operational modules can be implemented separately. The MILP models are generated and solved by the IBM ILOG CPLEX Optimizer (linear solver). The considered scenario 
Table 1. Outcomes in different scenarios.

\begin{tabular}{|c|c|c|c|c|c|c|c|c|}
\hline \multirow[b]{3}{*}{ Strategic planning modeling } & \multicolumn{8}{|c|}{ Strategy planning case study } \\
\hline & \multicolumn{2}{|c|}{$3 \mathrm{~s}$} & \multicolumn{2}{|c|}{$1 \mathrm{~S}$ and $2 \mathrm{~S}$} & \multicolumn{2}{|c|}{ MCC (cost based) } & \multicolumn{2}{|c|}{ MCC (distance based) } \\
\hline & GA & ILOG & GA & ILOG & GA & ILOG & GA & ILOG \\
\hline Active RDC & 4 & 4 & 4 & 4 & 4 & 3 & 11 & 14 \\
\hline Active CDC & 3 & 3 & 3 & 3 & 3 & 3 & 3 & 3 \\
\hline Active plants & 3 & 3 & 3 & 3 & 3 & 3 & 3 & 3 \\
\hline Total cost $(€)$ & $51,210,008$ & $52,103,008$ & $51,297,281$ & $52,386,112$ & $51,190,412$ & $52,626,312$ & $61,283,510$ & $64,316,780$ \\
\hline $\begin{array}{l}\text { Regional distribution cost } \\
\quad(€)\end{array}$ & $2,764,236$ & $2,932,438$ & $3,042,785$ & $3,128,231$ & $3,119,006$ & $3,358,781$ & $12,264,037$ & $13,652,115$ \\
\hline Distribution centre cost $(€)$ & $4,114,267$ & $4,381,247$ & $4,114,267$ & $4,381,247$ & $4,114,267$ & $4,381,247$ & $4,114,267$ & $4,381,247$ \\
\hline Plant handling cost $(€)$ & $16,996,219$ & $17,108,992$ & $16,996,219$ & $17,108,992$ & $16,996,219$ & $17,108,992$ & $16,996,219$ & $17,108,992$ \\
\hline $\begin{array}{l}\text { Average time to reach a } \\
\text { point of demand }(h)\end{array}$ & 63.41 & 65.61 & 55.81 & 57.23 & 61.94 & 64.12 & 61.76 & 63.54 \\
\hline
\end{tabular}

for the strategic model as described: multi-level (four levels), single period (e.g. 1 year), multi-product, capacitated in terms of handling at each level and production at the first one (the source level), multi-modal transportation. In the strategic planning, the distance between two generic locations are generally quantified adopting the Euclidean hypothesis, but the tool gives the user the option to measure the

exact road distance in quickest or shortest hypotheses. This is the mixed integer linear programming strategic planning model $3 \mathrm{~S}$, which can be obtained by the solver to find the optimal solution, or the solution as close as possible to the optimal when the instance of the decision problem is too wide and complex.

\section{Results and discussion}

In this case study, we consider a well-known manufacturing company made of three stages and four levels: COD: 318, RDCs: 15 , CDCs: 8 and number of production plant is 5 . In this model, we have tested a 6 months input data, in which six different products are introduced and sold in 25 countries. The customer demand is based on daily basis and acquired a single period strategic planning. A genetic algorithm is proposed to solve the aforementioned model and compare with ILOG CPLEX.

Table 1 shows the outcome obtained on the basis of strategy planning according to different scenarios. This is strategic planning for full optimization as shown in section 3.

ILOG CPLEX gives better result with compare to genetic algorithm, but computational time is more in all cases.

\section{Conclusion}

This paper presents a model to capture and design the real scenario of strategic planning for a logistics and supply chain network in order to minimize the fixed cost, transportation cost and handling cost associated with manufacturing plants, central and regional distribution centres. In the proposed model, the impact of manufacturers and cost associated to design the networks are considered by integrating the sourcing decisions into model. To encounter the problem complexity, two solution approaches are proposed: nature inspired genetic algorithm and ILOG CPLEX solver. A comparative study is done and quality of solution, where it is observed that ILOG CPLEX performs better than GA, but the computational time is vice versa.

\section{References}

[1] Pasandideh S H R, Niaki S T A and Asadi K 2015 Optimizing a bi-objective multi-product multi-period three echelon supply chain network with warehouse reliability. Expert Syst. Appl. 42(5): 2615-2623. doi:10.1016/j.eswa.2014.11. 018

[2] Wang F, Lai X and Shi N 2011 A multi-objective optimization for green supply chain network design. Decis. Support Syst. 51(2): 262-269. doi:10.1016/j.dss.2010.11.020

[3] He J, Huang Y, and Chang D 2014 Simulation-based heuristic method for container supply chain network optimization. Adv. Eng. Inform. doi:10.1016/j.aei.2014.08.001

[4] Kalaitzidou M A, Longinidis P and Georgiadis M C 2015 Optimal design of closed-loop supply chain networks with multifunctional nodes. Comput. Chem. Eng. 80: 73-91. doi:10.1016/j.compchemeng.2015.05.009

[5] Garg K, Kannan D, Diabat A and Jha P C 2015 A multicriteria optimization approach to manage environmental issues in closed loop supply chain network design. J. Cleaner Prod. 100: 297-314. doi:10.1016/j.jclepro.2015.02.075

[6] Pratap S, Daultani Y, Tiwari M K and Mahanty B 2015 Rule based optimization for a bulk handling port operations. $J$. Intell. Manuf. doi:10.1007/s10845-015-1108-7.

[7] Fattahi M, Mahootchi M, Govindan K and Moattar Husseini S M 2015 Dynamic supply chain network design with capacity planning and multi-period pricing. Transp. Res. E: Logist. Transp. Rev. 81: 169-202. doi:10.1016/j.tre.2015.06.007 
[8] Dejax P and Olivier P 2013 Sustainable supply chain network design: An optimization-oriented review. Omega 54: 1-48. doi:10.1016/j.omega.2015.01.006

[9] Mousavi S M, Alikar N, Niaki S T A and Bahreininejad A 2015 Optimizing a location allocation-inventory problem in a two-echelon supply chain network: A modified fruit fly optimization algorithm. Comput. Ind. Eng. 87: 543-560. doi:10.1016/j.cie.2015.05.022

[10] Kristianto Y, Gunasekaran A, Helo P and Hao Y 2014 A model of resilient supply chain network design: A two-stage programming with fuzzy shortest path. Expert Syst. Appl. 41(1): 39-49. doi:10.1016/j.eswa.2013.07.009

[11] Pramanik S, Jana D K, Mondal S K and Maiti M 2015 A fixed-charge transportation problem in two-stage supply chain network in Gaussian type-2 fuzzy environments. Inform. Sci. 325: 190-214. doi:10.1016/j.ins.2015.07.012

[12] Mousavia S M, Alikarb N, Niakic S T A and Bahreininejad A 2015 Optimizing a location allocation-inventory problem in a two-echelon supply chain network: A modified fruit fly optimization algorithm. 47: 543-560

[13] Simchi-Levi D, Kaminsky P, Simchi-Levi E and Shankar Ravi 2008 Designing and managing the supply chain: Concepts, strategies, and case studies. Tata McGraw-Hill Education

[14] Daultani Y, Kumar S, Vaidya O S and Tiwari M K 2015 A supply chain network equilibrium model for operational and opportunism risk mitigation. Int. J. Prod. Res. 53(18): 5685-5715 